\title{
Proposta di utilizzo di metodologie termografiche per il controllo di qualità di componenti meccanici
}

\author{
Carmelo Clienti, Guido La Rosa, Antonino Risitano \\ DIIM Dipartimento di Ingegneria Industriale e Meccanica, Università di Catania \\ Roberto D'Andrea \\ Magneti Marelli S.p.A. Exbaust System Industrial Management Technology, Venaria Reale (TO)
}

\begin{abstract}
RIASSUNTO. In base all'esperienza maturata in anni di sperimentazione sull'analisi delle caratteristiche meccaniche dei materiali mediante indagine termografica, nel presente lavoro è proposta una procedura per il controllo di qualità di componenti meccanici in linea di produzione, che è già stata argomento di brevetto.

Lo sviluppo di questo lavoro si colloca nell'ambito del progetto FIRB "Sistemi di produzione intelligenti, flessibili e riconfigurabili". L'attività svolta dal DIIM riguarda il controllo avanzato dell'affidabilità di componenti meccanici per l'industria automobilistica. Viene proposta, quindi, la realizzazione di una cella di controllo in linea di produzione capace di valutare la presenza di eventuali componenti difettosi attraverso l'analisi termica degli stessi, sollecitati secondo un modello predefinito.

L'attività, svolta in questa prima fase in laboratorio, è facilmente trasferibile in linea di produzione, considerando la possibilità di realizzare celle di prova in ambiente controllato, con condizioni praticamente identiche a quelle di laboratorio, eliminando gli effetti di disturbo che possono influenzare la risposta dell'indagine termografica in ambiente non strutturato.
\end{abstract}

ABSTRACT. Based on the experience of more than a quarter of century on the analysis of the endurance characteristics of materials using thermographic methodologies, the present paper deals on a proposal of a new procedure for the quality control on mechanical components in processing line. The procedure methodology was already patented by some of the authors.

The work described is included in the research programme FIRB Smartflex, particularly regarding the activity of the DIIM about the advanced control of the reliability of automotive elements and systems. A control cell, inside the processing line, able to evaluate the presence of defects in the automotive components by the application of defined cyclic loads and the analysis of the induced thermal maps, is proposed.

The results, in this first step found in laboratory, could be easily transferred to the processing line, considering the possibility of realizing a structured environment control cell, with ambient conditions very similar to those created in the laboratory, reducing the noise effects affecting the thermal data in a non-structured environment.

PAROle CHIAVE. Qualità strutturale; C.N.D.; Termografia; Analisi immagini.

\section{INTRODUZIONE}

$\mathrm{I}$ n oltre venticinque anni di attività di ricerca condotta dal gruppo di Progettazione Meccanica del DIIM sono state messe a punto nuove metodologie per la caratterizzazione statica e dinamica dei materiali con l'ausilio della termografia, attestati dalle numerose memorie scientifiche [1-16]. Il risultato di tali attività è stato fra l'altro un 
brevetto nazionale degli autori [17] del 1988 ed un successivo brevetto sempre degli stessi autori [18] di aggiornamento e di applicazione della metodologia. La potenzialità e l'applicabilità della metodologia termografica per la caratterizzazione dinamica di materiali è ormai riconosciuta come tecnica utilizzabile anche fuori dai laboratori al punto tale che altri studiosi stranieri, anche sulla base di quanto fatto precedentemente dal 1983 ad oggi dal gruppo di Catania (le osservazioni di base già presenti nei brevetti nazionali [17-18] vengono quasi integralmente riportate) hanno brevettato negli Stati Uniti nel 2009 [19-21] procedure idonee alla caratterizzazione sia di materiali che di componenti.

Le tecniche termografiche consentono la determinazione delle caratteristiche meccaniche attraverso l'osservazione dei transitori termici riconducibili agli effetti termoelastici e termoplastici dei materiali sottoposti a sollecitazioni statiche e/o dinamiche. Il rilievo termografico, inoltre, offrendo una visione a campo intero consente la determinazione della zona del componente che per prima subisce variazioni termiche riconducibili ad uno degli effetti sopracitati; tali transitori correlati con le cause che le hanno determinate consentono di stabilire se il componente rientra nelle indicazioni di progetto o se, al contrario, evidenzia un comportamento inatteso.

I vari studi del gruppo hanno portato ad una progressiva evoluzione della metodologia con una serie di variazioni originali, permettendo non soltanto la valutazione del limite di fatica ma anche quella dell'intera curva di fatica e delle condizioni di danneggiamento. Inoltre, la metodologia si è ulteriormente sviluppata per consentire prove ancora più rapide, per valutare la vita residua ed estendere la tecnica non solo ai materiali ma anche ai componenti o addirittura ai sistemi meccanici nelle peculiari condizioni di esercizio. L'esperienza maturata in laboratorio, in cui i parametri di prova sono più facilmente controllabili, ha suggerito agli autori di sperimentare l'applicabilità in ambienti confinati di non difficile controllo. Di conseguenza, si è estrapolato il ragionamento ai controlli di qualità di prodotti/processi, dove è possibile mettere a punto metodologie di controlli che si basano sul rilievo termografico, sfruttando le peculiarità di tale tecnica: assenza di invasività, tecnica a campo intero, parametri di prova ripetibili.

\section{OBIETTIVO DEL LAVORO}

Sulla base delle precedenti considerazioni, dopo avere sperimentato l'applicabilità del metodo di base su elementi di macchina in laboratorio e dopo avere verificato la possibilità della stima del danno [22] si è sviluppato un sistema per il monitoraggio non distruttivo di componenti meccanici in produzione che, come già detto, è stato oggetto di brevetto [18]. Utilizzando le indicazioni prima descritte, si giunge alla conclusione che, sottoponendo il componente meccanico o industriale ad un opportuno sistema di carico (che può essere quello di esercizio, di collaudo o derivato da questi) per un numero di cicli limitato (poche centinaia o migliaia secondo il componente stesso e la sua durata in esercizio), è possibile evidenziare, mediante analisi termica della superficie, se il componente presenta difetti strutturali che lo porteranno a rottura precoce già all'interno della linea stessa di produzione o immediatamente a valle di essa. Creando ambienti controllati ed operando mediante analisi differenziale di immagini, è possibile evitare errori che applicazioni in ambiente esterno potrebbero introdurre. Il controllo permetterà, quindi, di regolare i parametri di processo perché i componenti prodotti rientrino nel campo di tolleranza strutturale ammesso e/o di definire le caratteristiche strutturali dei vari lotti di produzione, in modo da poterne controllare i percorsi successivi. Utilizzando tale sistema come controllo di qualità è quindi possibile definire le non conformità del componente o della linea di produzione. In tal senso, quindi, il presente lavoro vuole essere un contributo efficace per la definizione di procedure per il controllo di qualità strutturale, possibilmente direttamente nei processi di produzione in linea.

La procedura adottata si articola secondo le seguenti attività.

Analisi del problema (determinazione delle specifiche, del target, della tempistica).

Definizione e scelta del componente.

Analisi delle condizioni di esercizio e definizione delle condizioni di carico e vincolo.

Campagna di prove in laboratorio per la definizione delle condizioni limiti.

Definizione delle mappe termiche di riferimento in ambiente controllato.

Campagna di prove all'interno della linea stessa di produzione o immediatamente a valle di essa in ambiente controllato con parametri identici a quelli del punto precedente.

Lo sviluppo di questo lavoro si colloca nell'ambito del progetto FIRB "Sistemi di produrione intelligenti, flessibili e riconfigurabili", che vede come partecipanti, oltre al DIIM dell'Università di Catania, il Centro Ricerche FIAT, l'IFAC-CNR di Firenze, il DIMEC dell'Università di Genova. Il progetto riguarda lo sviluppo di sistemi d'automazione orientati alla flessibilità in grado di soddisfare i requisiti di agile riconfigurabilità, adattabilità e riutilizzo delle soluzioni, favorendo la riduzione dei tempi e costi durante l'intero ciclo di vita. 
In questo quadro complesso, è stato definito dai partners del progetto, quale esempio di applicazione della metodologia di possibile controllo di qualità, lo sviluppo di una simulazione su una cella d'assemblaggio marmitte esistente in Magneti Marelli Exhaust System Industrial Management Technology, presso l'impianto di Venaria (TO). Il dispositivo oggetto di studio è una staffa sostegno tubazioni per sistemi di scarico automobilistico, che viene fissata sotto la vettura e che subisce, nel corso del proprio ciclo vita, sollecitazioni a fatica. Essa viene saldata con un processo MIG a filo continuo.

\section{DESCRIZIONE DELL'INDAGINE}

Direttamente dalla linea di produzione, è stata estratta una prima serie di componenti. La staffa è connessa al tubo tramite due cordoni di saldatura, si presenta con la medesima curvatura del tubo per l'intera lunghezza del cordone, per assumere successivamente una forma rettilinea. Il tratto di tubazione campione (Fig. 1) presenta una lunghezza complessiva di circa $75 \mathrm{~mm}$ e diametri esterno ed interno pari a $42 \mathrm{~mm}$ e $39 \mathrm{~mm}$ rispettivamente. Una seconda serie di componenti, di tipo diverso, presenta invece una lunghezza del tratto di tubazione di circa $220 \mathrm{~mm}$, definita a seguito delle indicazioni derivante dalle prime prove sperimentali, con gli stessi diametri del tubo (Fig. 2). La staffa è connessa al tubo da un solo lato tramite un cordone di saldatura; presenta inizialmente la medesima curvatura del tubo per tutto il tratto di saldatura, per poi assumere una doppia curvatura ed a seguire una forma rettilinea, in modo tale da aumentare, per esigenze applicative, l'eccentricità fra asse tubo ed asse staffa.

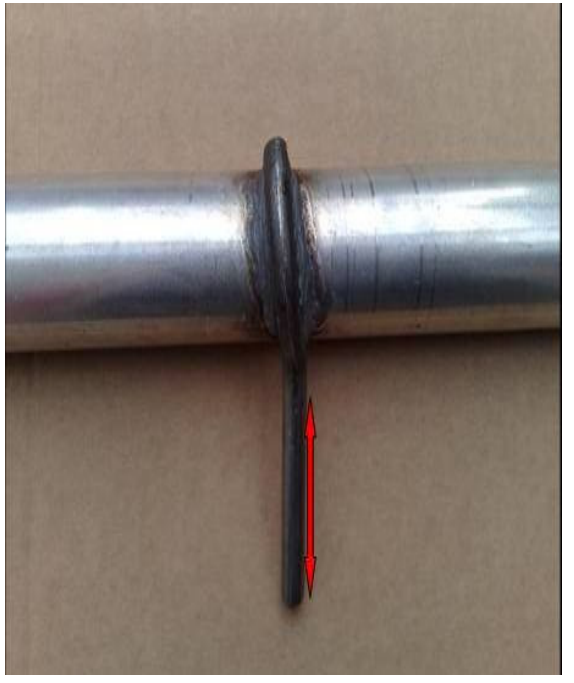

Figura 1: Prima serie di componenti

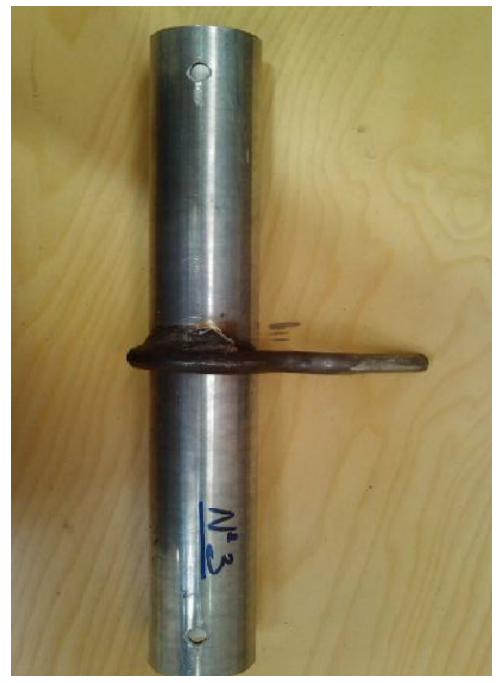

Figura 2: Seconda serie di componenti

In accordo con le indicazioni fornite da Magneti Marelli, entrambe le campionature sono state sollecitate, in questa prima fase, con forze agenti in direzione coassiale all'ultimo tratto della staffa.

Al fine di definire le caratteristiche della macchina di prova si è proceduto alla determinazione dell'ordine di grandezza della resistenza dell'intero componente (tubo-saldatura-perno) attraverso un calcolo approssimato della resistenza del cordone di saldatura. E' stato stimato che il carico di rottura della saldatura ad angolo per i componenti del primo tipo (doppio cordone di saldatura) è di circa $28 \mathrm{kN}$; di circa la metà per i componenti della seconda campionatura (un solo cordone di saldatura). Individuati gli ordini di grandezza delle condizioni di sollecitazione dei componenti, sono state scelte le macchine di prova: Instron 8501 configurata per prove assiali fino a $100 \mathrm{kN}$, per i test sui componenti della prima campionatura, e Instron 8872 , configurata per prove assiali fino a $25 \mathrm{kN}$, per i test su quelli della seconda campionatura.

E' stato necessario studiare, progettare e realizzare opportuni supporti che permettessero l'applicazione del carico desiderato. Relativamente alla prima tipologia di campioni, sono stati effettuati diversi studi preliminari di ancoraggi per collegare il componente alla Instron 8501 tra la cella di carico (in alto) e l'attuatore (parte inferiore della macchina di prova). In Fig. 3 si riportano la sequenza delle proposte vagliate e l'ancoraggio adottato (nella parte inferiore della figura), tralasciando per esigenze di spazio le considerazioni che ne hanno motivato la scelta. Le verifiche, analitica e mediante analisi FEM, hanno consentito la determinazione della più idonea geometria dell'ancoraggio (Fig. 4). Si evidenzia soltanto il fatto che la forma dei campioni non presenta alcun asse o piano di simmetria, e considerato che gli assi del perno e del tubo sono sghembi, si è proceduto allo studio di una struttura di ancoraggio che consentisse con manovre semplici la 
coincidenza tra l'asse della staffa, l'asse dell'attuatore e della cella di carico. La soluzione adottata consente di regolare il serraggio differenziale, attraverso quattro perni filettati, su una piastra in ERGAL solidale alla cella di carico, al fine di assicurare l'assialità tra staffa, attuatore e cella di carico.
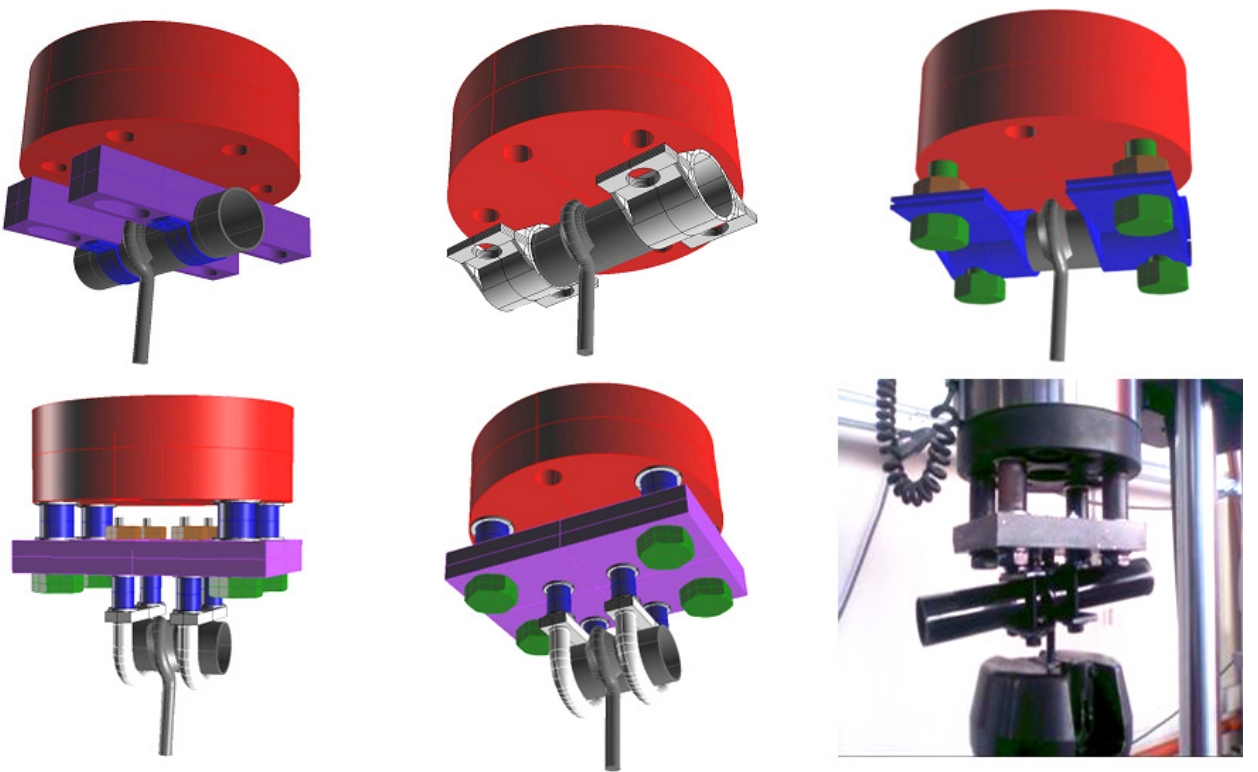

Figura 3: Studio preliminare sistemi di ancoraggio (in alto); ancoraggio adottato (in basso).
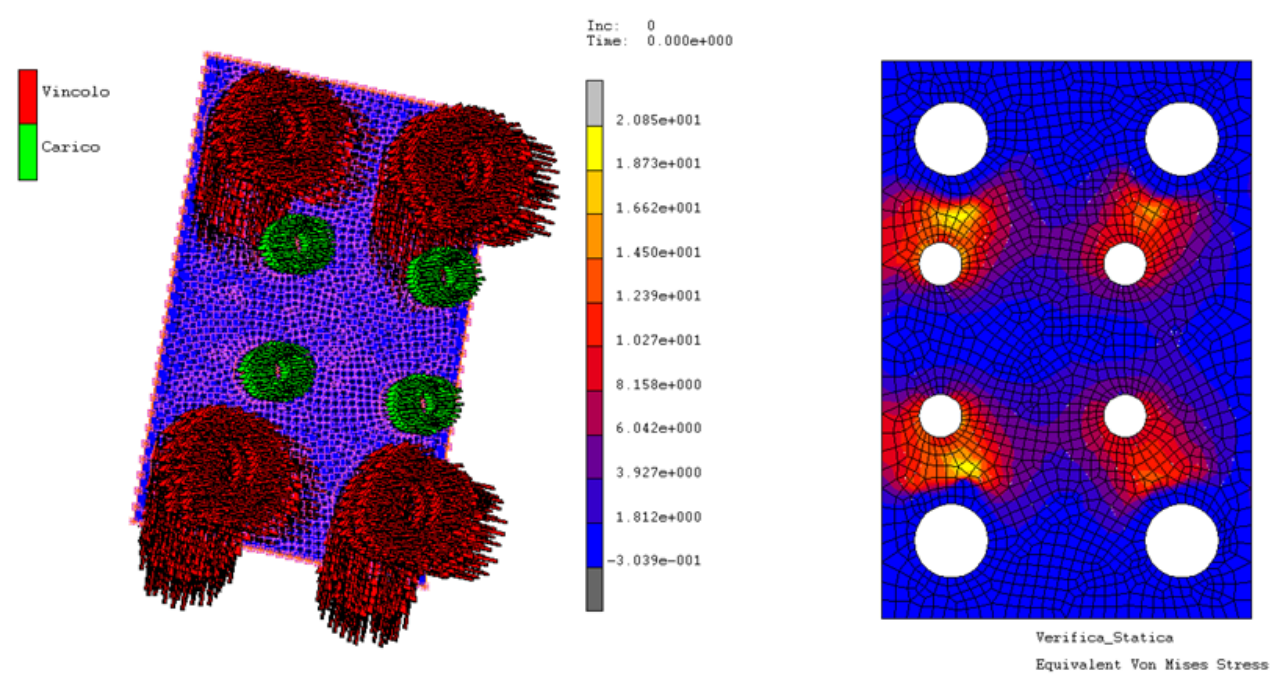

Figura 4: Schema per l'analisi FEM; Von Mises Stress.

Materiale isotropo, 2068 nodi, 1895 elementi quadrang. membrana 2D, spessore $25 \mathrm{~mm}$

A seguito dei risultati conseguiti con il primo set di prove (prima tipologia di campioni), per la seconda campionatura è stato studiato e realizzato un nuovo supporto, tale da ridurre gli effetti negativi evidenziati con la prima. In particolare, il secondo tipo di supporto consente di eliminare gli strisciamenti relativi fra provino e ancoraggio ed allontanare i vincoli dalla saldatura.

Riguardo il primo intento sono stati introdotti sopporti tipo Y SKF $(\mathrm{d}=40 \mathrm{~mm})$, così da permettere la rotazione attorno l'asse del tubo. Per il calettamento del cuscinetto e il tubo per via della differenza tra i diametri, è stato necessario l'ausilio di un elemento di riduzione.

Riguardo il secondo intento, i provini della seconda serie di componenti sono stati realizzati con una lunghezza del tubo di $220 \mathrm{~mm}$, contro i $75 \mathrm{~mm}$ dei precedenti. La piastra di supporto, sempre in ERGAL (Fig. 5), è stata modificata ed adattata rispetto alla prima per entrambe le macchine di prova. Analogamente al caso precedente, la verifica analitica e con FEA ha consentito la determinazione della geometria dell'ancoraggio (Fig. 6). 

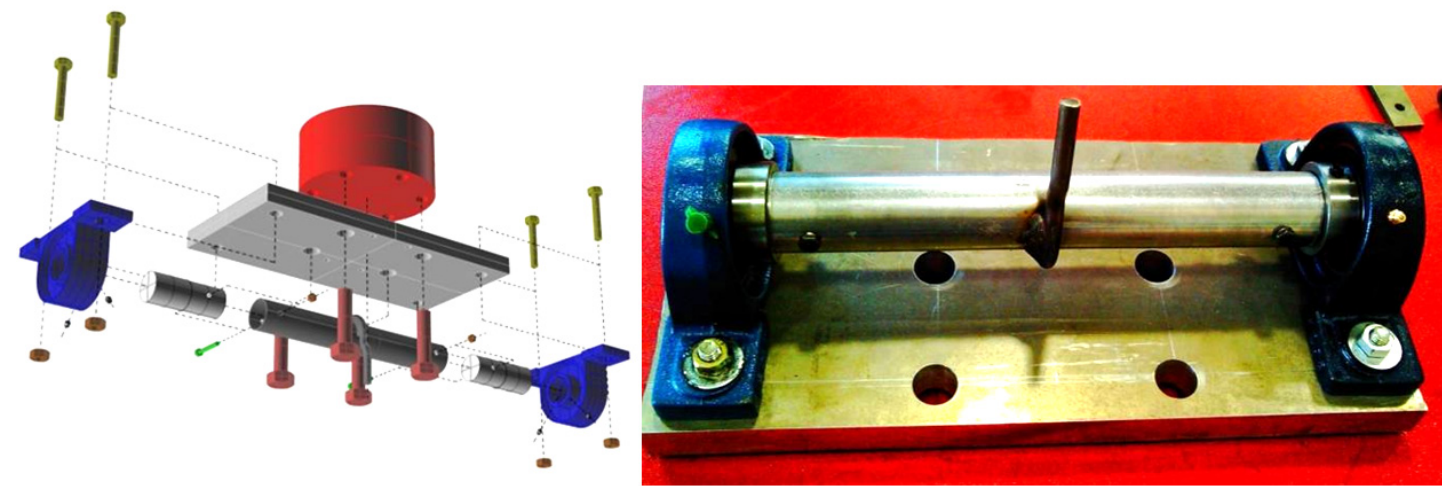

Figura 5: Modellazione piastra e supporto e sistema di vincolo del secondo tipo.

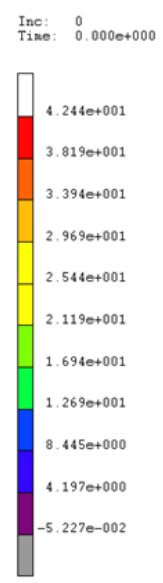

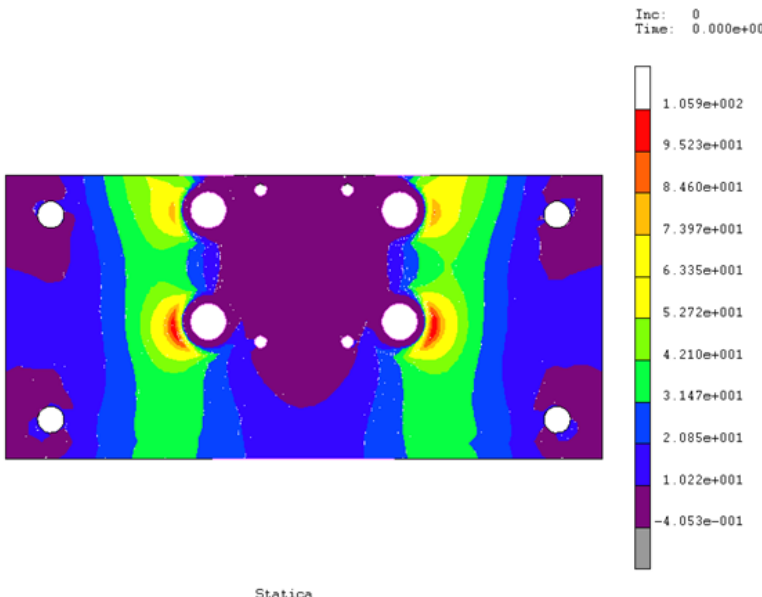

Statica
Equivalent Yon Mises Stress

(a)

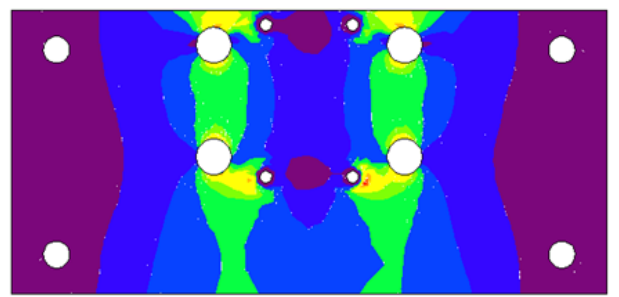

Statica

(b)

Figura 6: Von Mises Stress; (a) piastra su Instron 8501; (b) piastra su Instron 8872

Elementi membrana quadrangolare 2D; spessore 25mm; materiale isotropo.

Un'ulteriore indagine è stata condotta sullo stato di sollecitazione indotta sul componente, in particolare sulla staffa, per verificare quanto la sollecitazione fosse prossima alla monoassiale. Le simulazioni FEM evidenziano come la geometria del componente è tale da indurre sulla staffa una sollecitazione fortemente pluriassiale. In Fig. 7 si riportano i risultati della simulazione FEM.

I componenti della prima campionatura sono stati testati su macchina di prova Instron 8501 con il componente ancorato alla macchina di prova mediante gli ancoraggi di Fig. 3. Sono state condotte prove statiche e dinamiche. Le prime, volte alla ricerca del carico limite e del corrispondente transitorio termico (per l'eventuale ricerca della zona di transizione elastico-plastico); le seconde, per la definizione del carico limite dinamico e dei profili termici corrispondenti ai treni di carico applicato durante la prova di fatica.

Le prove statiche sono state eseguite in controllo di posizione con velocità $0.5 \mathrm{~mm} / \mathrm{min}$. La registrazione immagini termiche è stata effettuata ad 1 frame/s.

L'analisi dei rilievi termografici della prova evidenzia come non sia possibile estrapolare alcun dato significativo del comportamento dinamico. In Fig. 8 si riportano le condizioni del componente a fine prova: la rottura avviene sulla zona di saldatura con parziale strappo della staffa dal tubo, come si evidenzia anche sull'immagine termica del componente rilevata durante la prova.

La prova dinamica è stata eseguita con sollecitazione oscillante sinusoidale $(\mathrm{R}=-1)$, frequenza di oscillazione $15 \mathrm{~Hz}, 10.000$ cicli per ciclo di carico immagini termografiche 1 frame $/ 5 \mathrm{~s}$; con 14 blocchi di carico oscillante da $\pm 2 \mathrm{kN}$ a $\pm 15 \mathrm{kN}$. Dall'analisi dei dati termografici (variazioni di temperatura nel punto più caldo della superficie) si nota come, in corrispondenza del terzo blocco di carico (impostato a circa $\pm 4 \mathrm{kN}$ ) si ha un netto innalzamento della temperatura (freccia) su tutti gli spot sui quali è stata rilevata la variazione di temperatura. Tale valore di carico rappresenta il limite di 
fatica del componente nelle condizioni di vincolo adottate. Alla fine della prova si è notata un'ampia cricca situata sul bordo della saldatura.
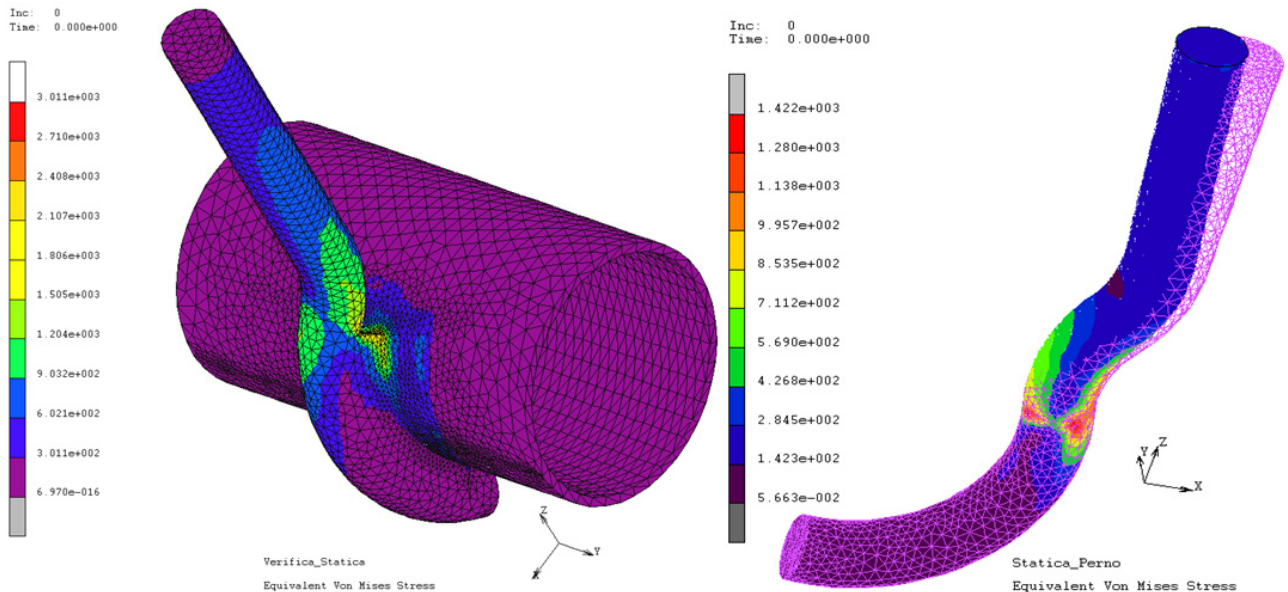

Figura 7: Simulazione della prova statica componente prima campionatura.

Simulazione 3D, funzione forma Tetraedo; 9955 nodi e 38605 elementi.
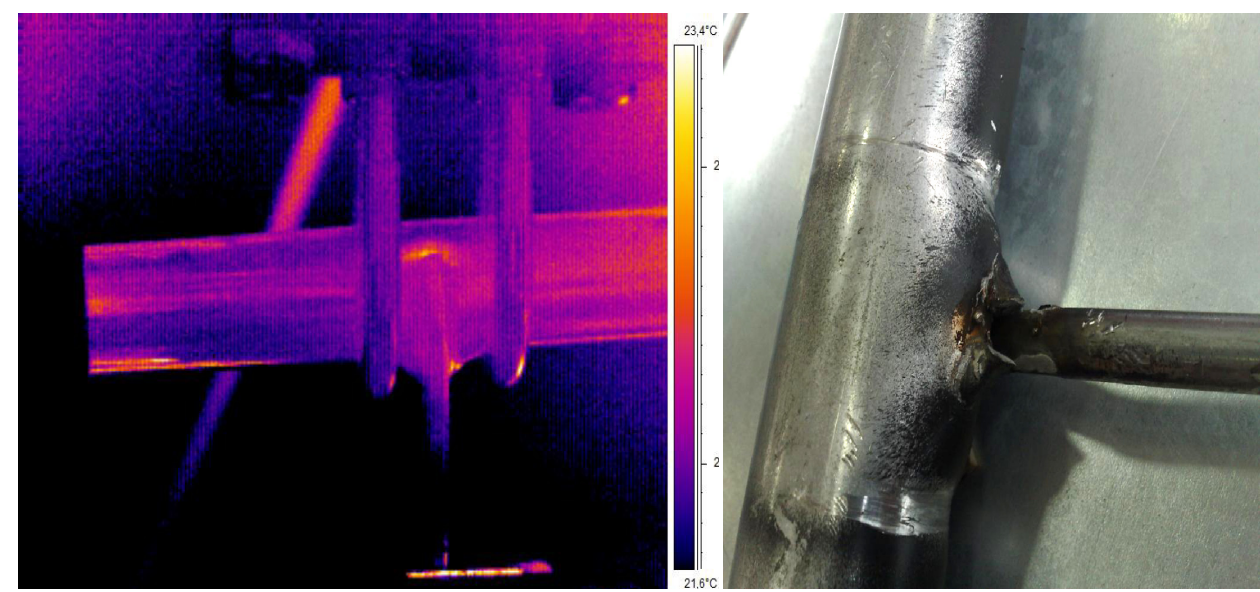

Figura 8: Immagine termica che evidenzia l'inizio della plasticizzazione nella zona di saldatura. Condizione del componente a fine prova.

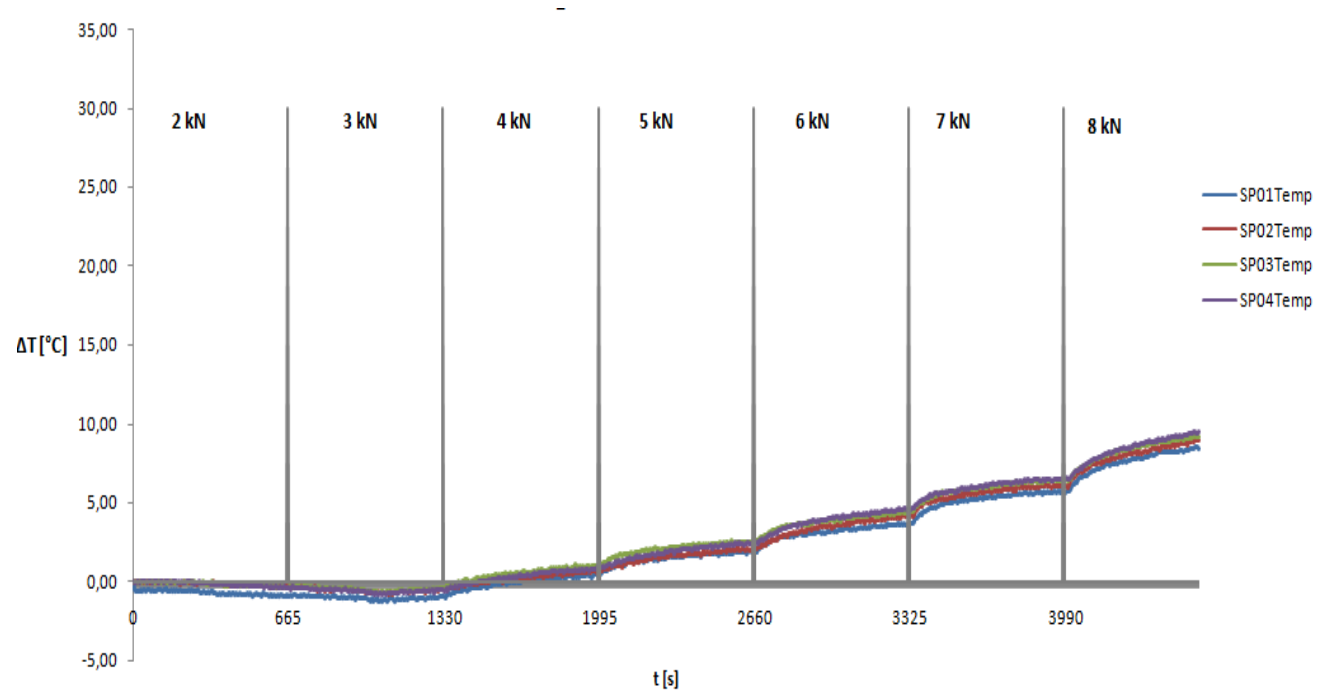

Figura 9: profilo termico per la prima serie di componenti. 
Le modalità di prova adottate per il test statico della seconda campionatura sono: macchina di prova Instron 8872 in controllo di posizione, velocità $2 \mathrm{~mm} / \mathrm{min}$, spostamento massimo $40 \mathrm{~mm}$; immagini termografiche rilevate ad 1 frame/5s. Dal diagramma di carico statico si osserva che a poco meno di $3 \mathrm{kN}$ si manifesta l'inizio della fase di plasticizzazione e a poco più di $8 \mathrm{kN}$ il cedimento del componente (Fig. 10). Tali indicazioni consentono di definire il protocollo della prova dinamica.

Le prove dinamiche per ogni componente testato sono state effettuate con sollecitazione pulsante sinusoidale $(\mathrm{R}=0)$ a frequenza $10 \mathrm{~Hz}$, con treni di carico di 10.000 cicli per step crescenti di $0.4 \mathrm{kN}$ (da $0.4 \mathrm{kN}$ a $3.2 \mathrm{kN}$ ) e rilevamento delle immagini termografiche ad 1 frame/5s. In Fig. 11 sono rappresentati i profili termici dei componenti provati. Dall'analisi si rileva che solo per sollecitazioni superiori al quarto livello $(1.6 \mathrm{kN})$ si hanno incrementi di temperatura significativi (praticamente diversi da zero). Il limite di fatica è stato fissato, quindi, in corrispondenza del terzo step di carico. Il risultato è stato confermato dalle successive prove dinamiche.

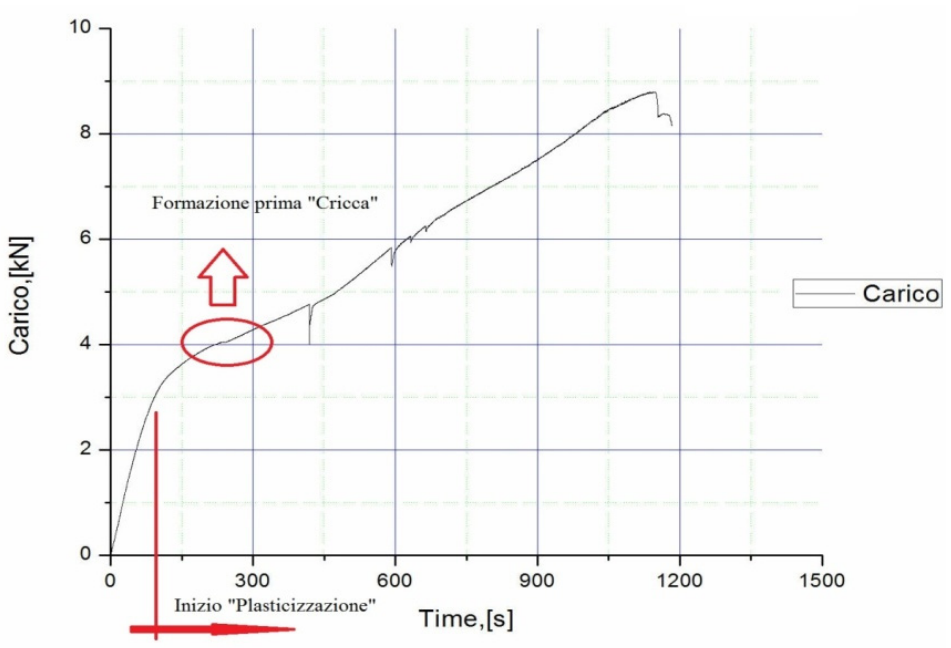

Figura 10: Prove statiche su componenti del secondo tipo.

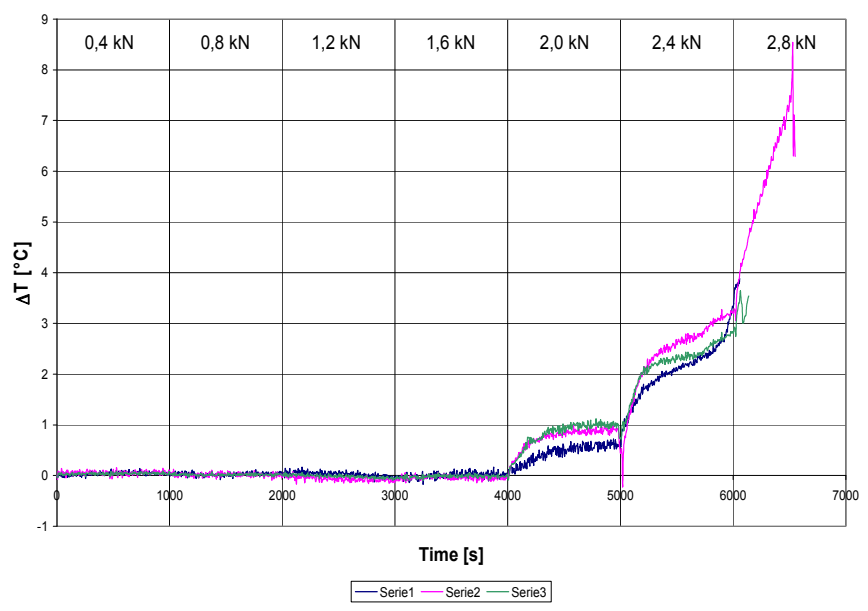

Figura 11: Profilo termico e limite di fatica in corrispondenza del quarto step di carico.

\section{DEFINIZIONE DELLE MAPPE TERMICHE DI RIFERIMENTO}

$\mathrm{S}$ ulla base dei risultati conseguiti, in particolar modo con le campagne di prova sulla seconda campionatura, è possibile definire il set di mappe termiche di riferimento per i controlli di qualità in linea. L'esperienza sperimentale prima riportata ha infatti evidenziato come in seno al componente, testato con sollecitazioni dinamiche di tipo pulsante, non si debbano manifestare riscaldamenti localizzati per treni di carico inferiori a quello corrispondente al carico 
di fatica. In tal modo, fissato un treno di impulsi di carico di valore inferiore al limite di fatica, nelle condizioni di carico e vincolo del componente esaminato, è possibile determinare la mappa termica di riferimento.

Per i componenti provati nel presente caso (Fig. 12) un componente passerebbe il controllo di qualità se, per una prova con le caratteristiche indicate in Tab. 1, la variazione di temperatura fosse del tipo di quella di riferimento e lo scarto fra valore misurato e di riferimento non superasse $0,2^{\circ} \mathrm{C}$. Il sistema potrebbe essere realizzato in automatico utilizzando sistemi di sottrazione d'immagini (immagine di prova meno mappa di riferimento) con valutazione automatica delle differenze termiche riscontrate.

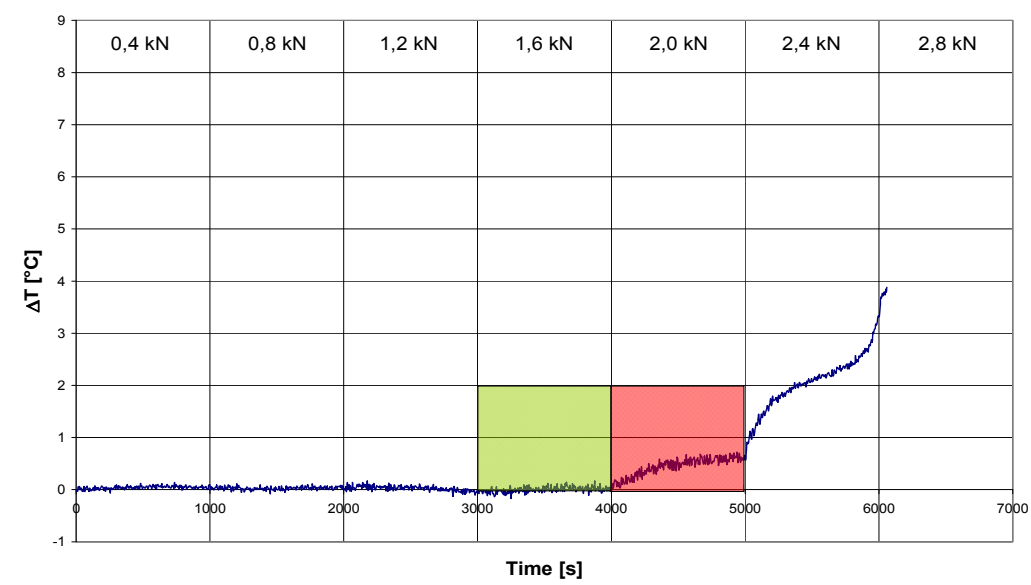

Figura 12: Valori di carico e variazione termica accettabili (verde) e non accettabili (rosso).

Per valutare in laboratorio l'attendibilità della metodologia, uno dei componenti è stato utilizzato come maschera termica, fornendo l'immagine di riferimento. La procedura è stata sviluppata in due fasi, la prima per evidenziare la possibilità di valutare il superamento del limite di fatica mediante analisi delle variazioni termiche, la seconda per verificare come un difetto possa essere rilevato dall'indagine termografica.

Per la prima fase è stato scelto un livello di carico significativamente più basso del limite di fatica, per il quale si mostrava sostanziale uniformità termica tra la zona di saldatura e la zona di appoggio. Il componente è stato prima testato ad un livello di carico $(1 \mathrm{kN})$ inferiore al limite di fatica $(1.6 \mathrm{kN})$, verificando che si trovava ancora in condizioni termoelastiche ( $\Delta \mathrm{T}$ negative), quindi, stressato a livelli di carico superiori al limite di fatica $(2 \mathrm{kN}$ e $2.3 \mathrm{kN}$ per 1000 cicli e $2.4 \mathrm{kN}$ per 2000 cicli) in modo da provocare la nucleazione della cricca e verificare la presenza di variazioni termiche crescenti col carico. Dopo ogni ciclo affaticante si è proceduto a verificare che il limite di fatica non fosse sceso sotto il livello di riferimento $(1 \mathrm{kN})$ mediante brevi cicli di carico (2000 o 1000 cicli) in cui si evidenziava ancora un comportamento termoelastico.

Successivamente, in relazione alla seconda fase, è stato valutato il nuovo limite di fatica (del componente criccato, abbassando il livello di carico da $1.6 \mathrm{kN}$ a $1.4 \mathrm{kN}$ fino a $1.3 \mathrm{kN}$,valore per cui l'incremento termico è risultato inferiore a $0.1^{\circ} \mathrm{C}$ e che si è assunto come nuovo limite di fatica (ovviamente inferiore al precedente a causa della cricca provocata). In questo modo, è stato sperimentalmente dimostrato come il limite di fatica si fosse ridotto e fosse possibile evidenziarlo con la tecnica termografica. Il componente, infine, è stato nuovamente sollecitato a valori elevati $(2.6 \mathrm{kN})$ e nuovamente portato alle condizioni di carico del nuovo limite trovato, mostrando stavolta un incremento termico di oltre $0.2^{\circ} \mathrm{C}$ nella zona di nucleazione della cricca. In questo modo si è verificato come l'immagine termica mostrasse l'indebolimento del componente a causa della propagazione della cricca. La sequenza di operazioni, mostrata in Fig. 12, dimostra come la metodologia permetta di valutare se il componente sia in grado di superare il test di qualità e come si possano evidenziare le variazioni del limite di fatica dovute all'affaticamento (o ad una cricca). La Fig. 14 mostra, infine, alcune immagini termiche relative alle varie fasi, ciascuna relativa ad ognuna delle dodici fasi riportate in Fig. 13.

Analoghe considerazioni permetterebbero di valutare se un componente sia in grado di resistere ad un definito numero di cicli. L'analisi a vita finita è ovviamente molto più complessa e delicata, ma la metodologia di controllo qualità è analoga: una maschera base da prendere come immagine di riferimento delle variazioni termiche ammesse per la vita finita prevista, da confrontare con l'immagine del pezzo in esame. In questo caso, diversamente dall'analisi d'immagini con carico sotto il limite di fatica, che non mostrano variazioni termiche dovute a microplasticizzazioni, infatti, ci sarebbero punti della mappa che presenterebbero un riscaldamento plastico, però limitato ad un prestabilito valore, secondo le indicazioni che si traggono dalle esperienze effettuate dagli autori sulla previsione di vita di provini e componenti e sul danno cumulativo $[11,13,15]$. 


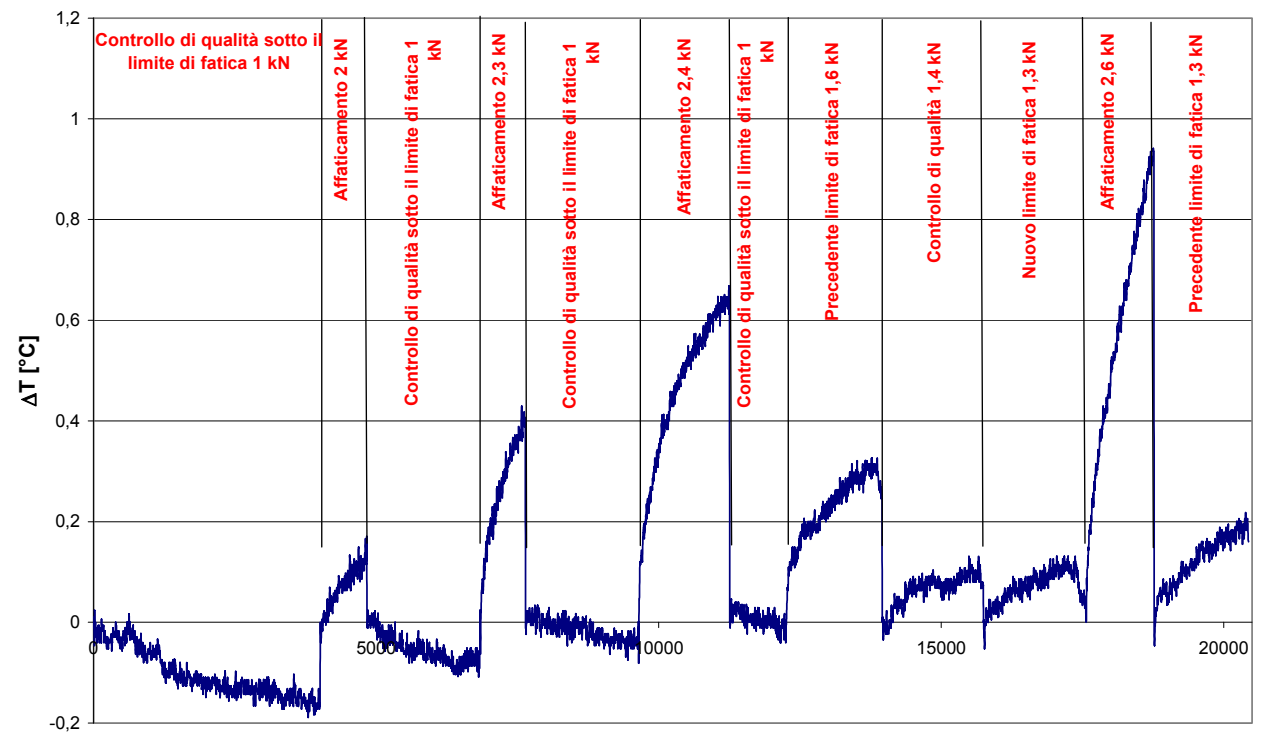

Cicli

Figura 13: Sequenza di operazioni affaticanti e di valutazione del limite di fatica.
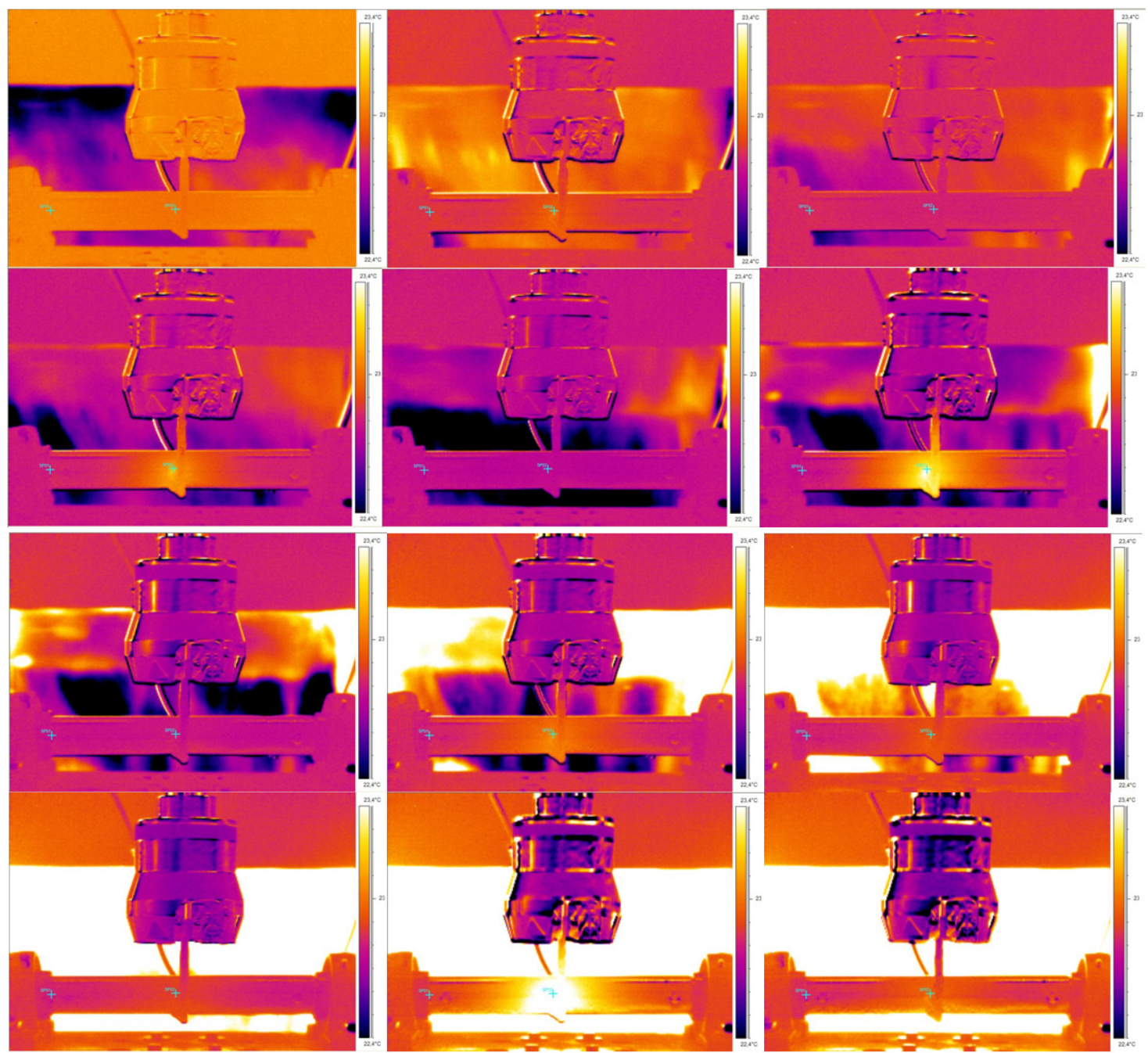

Figura 14: Immagini termiche relative alle dodici fasi di Fig. 13. 
Nella pratica applicazione, dopo avere realizzato sulla linea di produzione un ambiente adeguato (controllato) in cui le modalità di carico e le modalità di acquisizione possono essere effettuate come quelle prima descritte, viene definito il corrispondente protocollo di prova come in Tab. 1 (ad esempio facendo riferimento al secondo tipo di componente):

\begin{tabular}{llll}
\hline Tipologia del componente & $\mathrm{C} 2$ & Sensore termico & FLIR 3000 \\
Dimensioni: lunghezza & 220 & Emissività & 0.98 \\
Diametro esterno & 42 & Carico di prova & $1.6 \mathrm{kN}$ \\
Diametro interno & 39 & N cicli & 10000 \\
Macchina di prova & 8872 & Temperatura di riferimento & $20^{\circ} \mathrm{C}$ \\
Rapporto di carico & $\mathrm{R}=0$ & Mappa termica di riferimento & Immagine C2/16/5000 \\
Frequenza di prova & $5 \mathrm{~Hz}$ & Scarto di temperatura ammissibile & $+0.2^{\circ} \mathrm{C}$ \\
\hline
\end{tabular}

Tabella 1: Parametri di riferimento per il controllo di qualità.

Definita la mappa termica, il tratto di riferimento e le condizioni di prova, ciascun componente (o un certo numero se il controllo è di tipo statistico) deve essere sottoposto a controllo e per esso viene acquisita la corrispondente mappa termica, che viene confrontata con quella di riferimento.

\section{CONCLUSIONI}

L

o studio affrontato nel presente lavoro fa parte delle attività relative al progetto FIRB "Sistemi di produzione intelligenti, flessibili e riconfigurabili", che vede come partecipanti oltre al DIIM dell'Università di Catania, il Centro Ricerche FIAT, l'IFAC-CNR di Firenze, il DIMEC dell'Università di Genova. Il modulo affidato al gruppo di lavoro del Dipartimento di Ingegneria Industriale e Meccanica riguarda lo sviluppo di un efficace ed innovativo sistema di controllo strutturale di dispositivi di aggancio dei tubi di scarico di autovetture da alloggiare nella cella d'assemblaggio marmitte esistente in Magneti Marelli Exhaust System Industrial Management Technology, presso l'impianto di Venaria (TO). In particolare lo studio riguarda l'affidabilità di uno dei collegamenti, mediante saldatura MIG a filo continuo, del tubo di scarico alla autovettura che fissato sotto la vettura subisce, nel corso del proprio ciclo vita, sollecitazioni di fatica.

Estrapolando i risultati di sperimentazioni precedenti, e seguendo una procedura in precedenza brevettata, è stato proposto un sistema di controllo di qualità che attraverso l'analisi termografica del componente permette di decidere sulla sua affidabilità in condizioni di lavoro. La procedura prevede una sequenza di operazioni:

1. realizzazione di una cella di controllo in linea di produzione o alla uscita della fase produttiva attrezzata con sistema capace di produrre carichi variabili e adeguato sensore all'infrarosso termico;

2. caratterizzazione termodinamica del componente mediante la realizzazione di mappe termiche (variazione di temperatura-cicli per blocchi di carico) di prodotti integri;

3. definizione del profilo (tratto di mappa) di temperatura di riferimento per condizioni di carico prestabilite (carico, rapporto di carico R, frequenza di prova, numero di cicli) e definizione dello scarto di temperatura per l'accettazione del componente;

4. sistema di confronto (possibilmente automatico) del profilo di riferimento con quello osservato sul componente sotto indagine.

La procedura, che in esperienze di laboratorio ha dato risultati affidabili, è trasferibile anche in linea di produzione, in quanto è possibile realizzare una "cella di controllo" con condizioni simili a quelle realizzabili in laboratorio, svincolando il sistema da procedure di correzione dei parametri di ingresso e degli effetti di disturbo che in ambienti non strutturati potrebbero essere presenti.

\section{BIBLIOGRAFIA}

[1] T. Caltabiano, A. Geraci, M. Orlando, Il Progettista Industriale, 2 (1984).

[2] A. Geraci, G. La Rosa, A. Risitano, In: CRES Symposium, Catania (1984), pubblicato su ATA Ingegneria Automotoristica, 8 (1985) 665.

[3] G. Curti, G. La Rosa, M. Orlando, A. Risitano, In: XIV Congresso Nazionale AIAS, Catania, Italy, (1986).

[4] G. Curti, A. L.Geraci, A. Risitano, ATA Ingegneria Automotoristica, 10 (1989).

[5] A. Fichera, G. La Rosa, F. Patania, In: 44 Congresso Nazionale ATI, Cosenza, Italy, (1989). 
[6] A. L.Geraci, G. La Rosa, A. Risitano, In: VDI IMEKO/GESA Symposium, Düsseldorf, Germany, (1992).

[7] A. L. Geraci, G. La Rosa, A. Risitano, In: 15 th Polish Symposium on Experimental Mechanics, Warsaw, Poland, (1992).

[8] A. Geraci, G. La Rosa, A. Risitano, M. Grech, In: Digital Photogrammetry and Remote Sensing '95, St. Petersburg, Russia, SPIE, Series P, Vol. 2646, pp. 306-317, (1995).

[9] A. L. Geraci, G. La Rosa, A. Risitano, In: 7th ESIS International Conference on Mechanical Behaviour of Materials, The Hague, The Netherlands, (1995).

[10] E. Guglielmino, I. D.Guglielmino, G. La Rosa, A. Pasta, In: XXV AIAS International Conference on Material Engineering. Gallipoli- Lecce, (1996).

[11] G. La Rosa, A. Risitano, In: 17th Symposium on Experimental Mechanics of Solids, Warsaw, Poland, (1996) 498.

[12] G. Fargione, A. Geraci, G. La Rosa, A. Risitano, In: XXVII Congresso Nazionale AIAS , Perugia (1998).

[13] G. La Rosa, A. Risitano, International Journal of Fatigue, 22 (2000) 65.

[14] G. Fargione, A. Geraci, G. La Rosa, A. Risitano, International Journal of Fatigue, 24 (2002) 11.

[15] C. Clienti, G. Fargione, G. La Rosa, A. Risitano, G. Risitano, In: International Conference on Crack Paths CP2009, Vicenza, Italy (2009).

[16] A. Risitano, G. Risitano, In: Workshop IGF "Progettazione a fatica di giunzioni saldate (... e non). Sviluppi teorici e problemi applicativi”, Forni di Sopra (UD) (2009), pubblicato su Frattura ed Integrità Strutturale, 9 (2009) 113.

[17] A. Risitano, G. La Rosa, A. Geraci, Sistema di prova per la determinazione rapida del limite di fatica di materiali, Brevetto N. 1237378 (1993) su domanda del 28/11/1988.

[18] A. Risitano, G. La Rosa, Sistema di monitoraggio per la valutazione rapida della resistenza dinamica per materiali, componenti e sistemi meccanici in prova, esercizio e produzione, Brevetto N. 0001357456 (2009) su domanda del $18 / 05 / 2004$.

[19] Mehdi Amiri Darehbidi et al., Rapid determination of fatigue failure based on temperature evolution, Patent US 2009/0048788 Jul 31 (2008).

[20] M. Amiri, M. M. Khonsari, International Journal of Fatigue, 32 (2010) 382.

[21] M. Amiri, M. M. Khonsari, Life prediction of metals undergoing fatigue load based on temperature evolution, Material Science and Engineering A, in press (2009).

[22] G. Fargione, A. Geraci, L. Maiolino, A. Risitano, In: XXX Congresso Nazionale AIAS, Alghero (2001). 